\title{
Consecuencias de las reclamaciones judiciales sobre los médicos afectados.
}

Perea Pérez, Bernardo, Santiago Sáez, Andrés, Labajo González, Elena, Albarrán Juan, María Elena y Sánchez Sánchez, José Antonio.

Cita:

Perea Pérez, Bernardo, Santiago Sáez, Andrés, Labajo González, Elena, Albarrán Juan, María Elena y Sánchez Sánchez, José Antonio (2010). Consecuencias de las reclamaciones judiciales sobre los médicos afectados. Revista Clínica Española, 252, 1-6.

Dirección estable: https://www.aacademica.org/elenalabajogonzalez/14 ARK: https://n2t.net/ark:/13683/pcQr/NTt

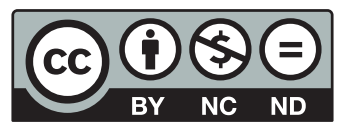




\title{
Revista Clínica Española
}

\section{ORIGINAL BREVE}

\section{Consecuencias de las reclamaciones judiciales sobre los médicos afectados}

\author{
B. Perea-Pérez, A. Santiago-Sáez, E. Labajo-González*, \\ M.E. Albarrán-Juan y J.A. Sánchez-Sánchez
}

Departamento de Toxicología y Legislación Sanitaria, Facultad de Medicina, Universidad Complutense de Madrid, Madrid, España

Recibido el 12 de abril de 2010; aceptado el 2 de agosto de 2010

\section{PALABRAS CLAVE \\ Demandas; \\ Reclamación judicial; \\ Resoluciones \\ judiciales; \\ Impactos; \\ Práctica profesional}

\section{KEYWORDS}

Claims;

Lawsuits;

Judgment;

Impacts;

Professional practice

\begin{abstract}
Resumen
Objetivos: Determinar las consecuencias que una reclamación judicial tiene para la vida personal y la práctica profesional de los médicos afectados.

Material y métodos: Estudio transversal, descriptivo, realizado sobre 169 encuestas cumplimentadas por médicos que han sido objeto de una reclamación judicial en España durante el período 2002-2005.

Resultados: La reclamación supuso una vivencia personal mala o muy mala en el $98,8 \%$. Un $85,1 \%$ reconoce una repercusión en su vida familiar. El $84 \%$ ha variado su forma de trabajar y el $57,4 \%$ reconoce repercusión en su vocación. En el $6 \%$ hubo resolución judicial desfavorable. Conclusiones: Se evidencian importantes repercusiones en los médicos después de una reclamación judicial, tanto en su vida personal y familiar, como en la forma de afrontar el ejercicio de la medicina.
\end{abstract}

(c) 2010 Elsevier España, S.L. Todos los derechos reservados.

\section{Consequences of lawsuits on affected doctors}

\begin{abstract}
Objectives: To determine the consequences of a judicial claim on the private life and professional practice of affected doctors.

Material and methods: A cross-sectional, descriptive study was performed on the basis of 169 surveys filled out by physicians subjected to a lawsuit in Spain in the period including 2002-2005. Results: The claim produced a bad or very bad personal experience in $98.8 \%$. A total of $85.1 \%$ admitted repercussions on family life, $84 \%$ made some change in the way they worked, and $57.4 \%$ admitted effects on their vocation. There was an unfavorable court ruling in $6 \%$. Conclusions: There were important repercussions on doctors after a lawsuit, both on their private and family life, as well as the way they approach medical practice.

(c) 2010 Elsevier España, S.L. All rights reserved.
\end{abstract}

* Autor para correspondencia.

Correo electrónico: elabajo@med.ucm.es (E. Labajo-González).

0014-2565/\$ - see front matter ๔ 2010 Elsevier España, S.L. Todos los derechos reservados. doi:10.1016/j.rce.2010.08.002 


\section{Introducción}

El incremento de las reclamaciones legales de los pacientes contra los médicos responsables de su asistencia es un hecho evidente en los últimos años. Las autoridades políticas sanitarias se han ocupado de proteger los derechos de los pacientes y han facilitado estas reclamaciones. Los gestores de hospitales y servicios clínicos, a su vez, tratan de determinar las circunstancias y maniobras clínicas que entrañan mayor riesgo para la seguridad de los pacientes.

Pocos trabajos se han preocupado de precisar las consecuencias que este aumento de la presión legal tiene en la vida personal y en la visión de la profesión de los médicos afectados. Además, estos escasos estudios existentes se centran sobre todo en los costes económicos directos que implican el aumento de la medicina defensiva y el incremento del coste de las pólizas de seguros de responsabilidad civil profesional ${ }^{1}$.

Está demostrada la aparición de medidas defensivas en la práctica de la medicina cuando el profesional o su entorno inmediato sufren una demanda legal ${ }^{2}$. Esta medicina defensiva incluye el abandono de ciertas prácticas profesionales técnicamente adecuadas por ser legalmente más peligrosas, y el aumento en la solicitud de pruebas complementarias con el único fin de aumentar la seguridad legal del médico ${ }^{3,4}$. Estas prácticas defensivas se documentan en todas las partes del mundo, incluso en países de diferente marco sociocultural ${ }^{5}$.

Apenas existen estudios que se centren en las repercusiones más personales que estas demandas puedan implicar para los médicos afectados ${ }^{6}$ o para su entorno familiar ${ }^{7}$. Algunos estudios recogen la existencia de una alta tasa de abandono de la actividad asistencial en las especialidades legalmente más reclamadas, e incluso el traslado a zonas donde la presión de los pacientes y el marco legal no son $\tan$ estrictos ${ }^{8-10}$.

El objetivo de este trabajo se centra en estimar las repercusiones que una reclamación judicial (civil o penal) tiene en los médicos implicados, tanto en la esfera profesional como en la personal.

\section{Material y métodos}

Se realizó un análisis transversal de la información recogida mediante cuestionarios específicos. Para el estudio se seleccionaron los médicos de todo el territorio español y de todas las especialidades con póliza individual de responsabilidad civil profesional en la Agrupación Mutual Aseguradora (AMA), que hubiesen sido reclamados judicialmente durante el período 2002-2005, y cuyo expediente estuviese cerrado (mediante sentencia judicial o acuerdo extrajudicial). Los datos personales de los médicos seleccionados han sido manejados únicamente por sus depositarios. Los autores de este estudio únicamente han tenido acceso y han trabajado con datos disociados.

Se seleccionaron 699 expedientes correspondientes a otros tantos médicos que cumplían estos requisitos, y se les envió un cuestionario confeccionado específicamente (tabla 1). El cuestionario era anónimo y se precedía por una carta en la que se detallaban los objetivos del estudio y quienes lo iban a realizar. El cuestionario constaba de 29 ítems agrupados en cinco bloques: perfil del profesional demandado (edad, sexo, especialidad, existencia de demandas previas), características y circunstancias de la reclamación judicial (ámbito de asistencia, ámbito judicial, resultado, etc.), actitud y comportamiento del paciente y del entorno del profesional, consecuencias personales y profesionales que la reclamación ha tenido sobre el médico demandado (sobre su forma de trabajar, sobre su visión de la profesión, sobre su relación con los pacientes, etc.) y otras cuestiones generales relativas a conocimientos médico-legales.

La encuesta fue remitida a los médicos seleccionados por el Departamento de Responsabilidad Profesional de la AMA, y fue dicho departamento el que recibió las respuestas.

Las variables principales fueron los daños al paciente, el tipo de demanda, la vivencia personal, el abandono del trabajo, la toma o no de precauciones legales ulteriores, la auto-consideración como responsable y la forma de relacionarse posteriormente con los pacientes. Se consideraron variables secundarias a sexo, grupo de edad, especialidad médica, comportamiento del paciente, ámbito sanitario, afectación de la forma de trabajar, valoración y resto de preguntas del cuestionario.

Estadísticamente se realizó un análisis descriptivo de frecuencias de las variables contempladas en el estudio. Posteriormente se compararon algunas de estas variables mediante el cruce de tablas de contingencia Chi cuadrado.

\section{Resultados}

Respondieron al cuestionario 169 de los 699 médicos a los que fue remitido (25,26\%). De los 169 cuestionarios examinados correspondientes a médicos demandados judicialmente, $122(76,7 \%)$ eran hombres y $37(23,3 \%)$ a mujeres, 10 cuestionarios no reflejan el sexo. El grupo de edad más representado fue el comprendido entre los 40 y 49 años $(41,3 \%)$.

En la tabla 2 se indican las especialidades más reclamadas así como el daño producido, el tipo de demanda y la resolución judicial. En la mayoría de los casos (98 casos, $58 \%$ de los casos) la demanda se dirigió contra el médico y el centro sanitario, mientras que en un $42 \%$ de los casos (71) se dirigió exclusivamente contra el médico. En el 80,2\% de los casos (134 ocasiones) el médico afectado no había tenido ningún problema legal previo con pacientes. En lo referente al ámbito sanitario en el que se produjo la asistencia demandada, en 96 casos $(56,8 \%)$ fue en el ámbito de la medicina privada y en 52 casos $(30,8 \%)$ en la medicina pública.

En 147 ocasiones (87\%) el médico no se consideraba responsable de lo ocurrido, en 19 casos $(11,25 \%)$ responsable parcial, y en 3 casos $(1,8 \%)$ totalmente responsable. La vivencia personal de la situación en el momento de producirse fue "muy mala", en 128 casos (76,2\%), "mala"' en 38 $(22,6 \%)$ e "indiferente" en 2 (1,2\%).

El $81 \%$ de los encuestados (136 casos) no dejó de trabajar por estos hechos, 24 médicos (14,3\%) dejaron de trabajar algún día y $8(4,8 \%)$ no acudieron a trabajar durante más de una semana. El comportamiento del paciente, o de su familia, fue calificado de "muy malo"' por el 46,1\% (77 casos) y "malo" por el 25,1\% (42 casos). 
Tabla 1 Cuestionario de reclamaciones a médicos

\begin{tabular}{|c|c|}
\hline Preguntas & Respuestas \\
\hline ¿Qué tipo de demanda o reclamación sufrió usted? & $\begin{array}{l}\text { Judicial penal } \\
\text { Judicial civil } \\
\text { Judicial contencioso-administrativa } \\
\text { Judicial penal + judicial civil } \\
\text { Otros }\end{array}$ \\
\hline ¿Ha tenido algún otro tipo de problema legal con los pacientes? & $\begin{array}{l}\text { No } \\
\text { Sí }\end{array}$ \\
\hline $\begin{array}{l}\text { ¿La reclamación que nos ocupa fue dirigida a usted sólo o afectó a más } \\
\text { profesionales? }\end{array}$ & $\begin{array}{l}\text { Sólo a mi } \\
\text { Conjunta con otros }\end{array}$ \\
\hline ¿Con qué área o especialidad de la medicina se relaciona? & $\begin{array}{l}\text { Ginecología y obstetricia } \\
\text { Traumatología y cirugía ortopédica } \\
\text { Cirugía plástica y reparadora } \\
\text { Oftalmología } \\
\text { Otras especialidades quirúrgicas } \\
\text { Otras especialidades médicas } \\
\text { Medicina extrahospitalaria }\end{array}$ \\
\hline ¿Público o privado? & $\begin{array}{l}\text { Público } \\
\text { Privado } \\
\text { Ambos }\end{array}$ \\
\hline ¿En qué ámbito ocurrió? & $\begin{array}{l}\text { Consulta de Medicina Atención Primaria } \\
\text { Consulta de especialidades } \\
\text { Urgencias (en todas sus modalidades) } \\
\text { Quirófano } \\
\text { Paritorio } \\
\text { Atención hospitalaria general } \\
\text { Otros }\end{array}$ \\
\hline ¿Qué daños dijo el paciente haber sufrido? & $\begin{array}{l}\text { Muerte } \\
\text { Otras secuelas }\end{array}$ \\
\hline ¿Considera que tuvo algún tipo de responsabilidad en lo ocurrido? & $\begin{array}{l}\text { No } \\
\text { Parcialmente } \\
\text { Sí }\end{array}$ \\
\hline ¿Está terminado el asunto? & No \\
\hline En el caso de estar terminado ¿cómo lo hizo? & $\begin{array}{l}\text { Arreglo extrajudicial } \\
\text { Absolución } \\
\text { Condena } \\
\text { Otros }\end{array}$ \\
\hline ¿Tuvo problemas con el Seguro de Responsabilidad Civil? & $\begin{array}{l}\text { No } \\
\text { Sí (especifique cuál) }\end{array}$ \\
\hline ¿Tuvo problemas con la dirección sanitaria o la inspección del propio centro? & $\begin{array}{l}\text { No } \\
\text { Sí (especifique cuál) }\end{array}$ \\
\hline ¿Cómo se sintió cuando recibió la demanda? & $\begin{array}{l}\text { Muy mal } \\
\text { Mal } \\
\text { Indiferente }\end{array}$ \\
\hline ¿Cuál fue el comportamiento del paciente? & $\begin{array}{l}\text { Muy malo } \\
\text { Malo } \\
\text { Neutro/aceptable } \\
\text { No lo sabe }\end{array}$ \\
\hline ¿Dejó de trabajar por esta causa en algún momento? & $\begin{array}{l}\text { No } \\
\text { Algún día } \\
\text { Más de una semana }\end{array}$ \\
\hline $\begin{array}{l}\text { ¿Cree que varió técnicamente su forma de trabajar en aquella época (número } \\
\text { de pruebas complementarias, prácticas defensivas, etc.)? }\end{array}$ & $\begin{array}{l}\text { Nada } \\
\text { Algo } \\
\text { Claramente sí }\end{array}$ \\
\hline ¿Tuvo este asunto alguna repercusión en su vida personal o familiar? & $\begin{array}{l}\text { Ninguna } \\
\text { Alguna } \\
\text { Claramente sí }\end{array}$ \\
\hline
\end{tabular}


Tabla 1 (Continuación)

\begin{tabular}{|c|c|}
\hline Preguntas & Respuestas \\
\hline $\begin{array}{l}\text { ¿Cree que aquel asunto ha tenido alguna repercusión en su forma de trabajar } \\
\text { actual? }\end{array}$ & $\begin{array}{l}\text { Ninguna } \\
\text { Alguna } \\
\text { Claramente sí }\end{array}$ \\
\hline ¿Y en su vocación? & $\begin{array}{l}\text { Ninguna } \\
\text { Alguna } \\
\text { Claramente sí }\end{array}$ \\
\hline ¿Y en la forma de relacionarse con los pacientes en la actualidad? & $\begin{array}{l}\text { Ninguna } \\
\text { Alguna } \\
\text { Claramente sí }\end{array}$ \\
\hline ¿Toma ahora más precauciones legales (consentimiento informado, más & No \\
\hline pruebas complementarias, seguro de Responsabilidad Civil, etc.)? & Sí (especifique cuáles) \\
\hline ¿Cree que su formación médico-legal fue suficiente entonces? & $\begin{array}{l}\text { No } \\
\text { Sí }\end{array}$ \\
\hline ¿Y cree que lo es ahora? & $\begin{array}{l}\text { No } \\
\text { Sí }\end{array}$ \\
\hline ¿Tiene usted algún conocimiento de la Ley 41/2002, Básica Reguladora de la & Sí \\
\hline Autonomía del Paciente? & $\begin{array}{l}\text { Sólo de forma superficial } \\
\text { No }\end{array}$ \\
\hline ¿Considera que los sistemas de asistencia sanitaria, públicos o privados, están & No \\
\hline preparados para el incremento de las reclamaciones legales por parte de los & Sí \\
\hline 6aaimathese produjo el problema, ¿cree usted que podría haber manejado mejor & No \\
\hline \multicolumn{2}{|l|}{$\begin{array}{l}\text { ¿Quiere añadir algún comentario u observación más? } \\
\text { Datos estadísticos del profesional }\end{array}$} \\
\hline \multirow[t]{2}{*}{ Sexo } & Hombre \\
\hline & Mujer \\
\hline \multirow[t]{4}{*}{ Edad cuando se produjo la demanda } & $20-29$ \\
\hline & $30-29$ \\
\hline & $40-49$ \\
\hline & 50 o más \\
\hline
\end{tabular}

La repercusión de la demanda en la vida familiar del médico fue muy importante en 69 ocasiones $(41,1 \%)$, importante en $74(44 \%)$ y nula en $25(14,9 \%)$.

Ciento quince médicos $(68 \%)$ consideran haber tomado más precauciones legales tras la demanda. Setenta y cuatro médicos $(43,8 \%)$ piensan que la demanda ha generado modificaciones importantes en su forma de trabajar, 68 (40,2\%) reconocen alguna variación y el 16\% (27 casos) no ha modificado su forma de trabajar. En cuanto a la forma de relacionarse con el paciente, 64 médicos $(38,1 \%)$ contestan que "claramente si" ha variado, $67(39,9 \%)$ reconocen "alguna repercusión"' y 37 (22\%) "ninguna". Respecto a su vocación médica, 40 respuestas $(23,7 \%)$ reconocen una variación clara a raíz del problema legal, 57 (33,7\%) una influencia relativa y 72 casos $(42,6 \%)$ no reconoce ninguna influencia (tabla 2).

El análisis Chi cuadrado muestra algunos datos interesantes. Existe una relación estadísticamente significativa (21/37 mujeres, $56,7 \%$, frente a 48 casos/122 varones, $39,3 \%, p<0,01)$ entre el hecho de ser mujer y la existencia de una clara repercusión en la vida personal y familiar del médico. También existe relación entre el fallecimiento del paciente y la vivencia del suceso $(35 / 38,92,1 \%$, de los casos con fallecimiento se sintieron "muy mal", $3 / 38$ se sintieron "mal, y ninguno se sintió "indiferente").

\section{Discusión}

Las repercusiones que una reclamación judicial tiene sobre el médico afectado es un tema muy poco estudiado, no existiendo estudios comparables con el presente en la bibliografía.

Sobre el tamaño muestral queremos reseñar la extraordinaria dificultad que entraña conseguir el acceso a los datos, muy dispersos y protegidos tanto por entidades públicas como privadas. Por otra parte, es de mencionar el bajo número de médicos que ha respondido al cuestionario $(25,26 \%)$. Consideramos que a esta cuestión ha debido contribuir el hecho de que para muchos profesionales no es agradable rememorar una experiencia como la de "sentarse en un banquillo".

Parece especialmente significativo que la mayoría de los médicos participantes (87\%) no se considere responsable de los hechos que motivaron la demanda. Este hallazgo parece poco acorde con la realidad percibida en los tribunales, donde raramente la razón la tiene exclusivamente una de las partes. No obstante no dudamos de la sinceridad de estas respuestas ya que está contenida en encuestas anónimas. Consideramos este dato como uno de los más importantes en la interpretación de los cuestionarios. Muestra de lo anterior es que el $71,2 \%$ describe como malo o muy malo el 
Tabla 2 Características de las demandas presentadas, de los médicos a los que se dirige y consecuencias de las demandas sobre los médicos (porcentajes e incidencias)

\begin{tabular}{|c|c|c|c|}
\hline \multicolumn{2}{|c|}{ Características de los médicos demandados y de la demanda } & \multicolumn{2}{|l|}{ Repercusiones sobre los médicos demandados } \\
\hline Sexo & & Se considera responsable & \\
\hline Hombre & $122(76,7 \%)$ & No & 147 (87\%) \\
\hline Mujer & $37(23,3 \%)$ & Parcial & $19(11,25 \%)$ \\
\hline No contestan & 10 & Sí & $3(1,8 \%)$ \\
\hline \multicolumn{2}{|l|}{ Grupos de edad } & \multicolumn{2}{|l|}{ Vivencia personal } \\
\hline $20-29$ & $4(2,5 \%)$ & Muy mala & $128(76,2 \%)$ \\
\hline $30-39$ & $42(26,25 \%)$ & Mala & $38(22,6 \%)$ \\
\hline $40-49$ & $66(41,25 \%)$ & Indiferente & $2(1,2 \%)$ \\
\hline$\geq 50$ & $48(30 \%)$ & No contestan & 1 \\
\hline No contestan & 9 & & \\
\hline \multicolumn{2}{|l|}{ Especialidades } & \multicolumn{2}{|l|}{ Dejó de trabajar } \\
\hline Ginecología y Obstetricia & $35(21,2 \%)$ & No & 136 (81\%) \\
\hline Cirugía plástica & $29(17,6 \%)$ & Algún día & $24(14,3 \%)$ \\
\hline Oftalmología & $13(7,9 \%)$ & $>1$ semana & $8(4,8 \%)$ \\
\hline Otros & $92\left(46^{\prime} 7 \%\right)$ & No contestan & 1 \\
\hline \multicolumn{2}{|l|}{ Daños al paciente } & \multicolumn{2}{|l|}{ Comportamiento paciente } \\
\hline Muerte & $38(23,2 \%)$ & Muy malo & $77(46,1 \%)$ \\
\hline Secuelas & $126(76,8 \%)$ & Malo & $42(25,1 \%)$ \\
\hline No contestan & 5 & Otros & $50(28,8 \%)$ \\
\hline \multicolumn{2}{|l|}{ Tipo de demanda } & \multicolumn{2}{|l|}{ Precauciones legales } \\
\hline Penal & $89(53,6 \%)$ & Sí & $115(68 \%)$ \\
\hline Civil & $46(27,7 \%)$ & No & $54(32 \%)$ \\
\hline Otros & $34(18,7 \%)$ & & \\
\hline \multicolumn{2}{|l|}{ Resolución } & \multicolumn{2}{|l|}{ Afectó a la vida } \\
\hline Absuelto & $69(41,6 \%)$ & Muy importante & $69(41,1 \%)$ \\
\hline Arreglo extrajudicial & $9(5,4 \%)$ & Importante & $74(44 \%)$ \\
\hline Condena & $10(6 \%)$ & Nula & $25(14,9 \%)$ \\
\hline Otros & $81(47 \%)$ & No contestan & 1 \\
\hline \multicolumn{2}{|l|}{ Dirigida contra } & \multicolumn{2}{|l|}{ Forma de trabajar } \\
\hline Sólo el médico & $71(42 \%)$ & Claras & $74(43,8 \%)$ \\
\hline \multirow[t]{2}{*}{ En conjunto } & $98(58 \%)$ & Alguna & $68(40,2 \%)$ \\
\hline & & No & $27(16 \%)$ \\
\hline \multicolumn{2}{|l|}{ Ámbito sanitario } & \multicolumn{2}{|l|}{ Vocación } \\
\hline Público & $52(30,8 \%)$ & Claramente sí & $40(23,7 \%)$ \\
\hline Privado & $96(56,8 \%)$ & Alguna & $57(33,7 \%)$ \\
\hline \multirow[t]{6}{*}{ Otros } & $21(12,4 \%)$ & Ninguna & $72(42,6 \%)$ \\
\hline & & \multicolumn{2}{|l|}{ Forma de relacionarse con futuros pacientes } \\
\hline & & Claramente sí & $64(38,1 \%)$ \\
\hline & & Alguna & $67(39,9 \%)$ \\
\hline & & Ninguna & $37(22 \%)$ \\
\hline & & No contestan & 1 \\
\hline
\end{tabular}

comportamiento del paciente o de su familia, y la vivencia personal del médico es descrita por ellos como muy mala en el $76,2 \%$, y mala en el $22,6 \%$ de los casos. Estos porcentajes son esperables si tenemos presente que, como señalamos anteriormente, el $87 \%$ de los médicos que han respondido no creen haber obrado incorrectamente. El $85,1 \%$ reconoce alguna repercusión en su vida familiar coincidiendo con otros estudios $^{7}$. Resulta llamativa la existencia de una relación muy significativa $(p<0,01)$ entre el hecho de que el reclamado sea mujer y la existencia de una clara repercusión en su vida personal y familiar.
En la gran mayoría de casos, el médico se siente atacado debido a unos hechos sobre los que no reconoce responsabilidad y que tienen para él importantes consecuencias. También parece evidente que el hecho de haber sufrido un proceso judicial implica importantes cambios en la forma de trabajar y en la visión de la profesión. El 68\% afirma tomar más precauciones legales, el $84 \%$ dice que el episodio le ha hecho variar su forma de trabajar, y el $78 \%$ que ha cambiado su forma de relacionarse con los pacientes. Estos datos coinciden con los de otros estudios que señalan la aparición de prácticas defensivas en más del $90 \%$ de los médicos 
afectados por problemas de este tipo ${ }^{4}$. Incluso un $57,4 \%$ de los médicos participantes en nuestro estudio refiere que este suceso ha tenido clara repercusión en su vocación médica.

En nuestra opinión estos datos evidencian las importantes repercusiones profesionales que implica esta situación. Y ello teniendo en cuenta que en el presente estudio sólo en el $6 \%$ de los casos se ha producido condena judicial.

La conclusión de este trabajo evidencia algo sospechado por todos los profesionales que nos relacionamos con el tema. El aumento de la presión legal sobre los médicos, sin entrar en sus causas, tiene efectos que a menudo son ignorados a pesar de su trascendencia. Los médicos afectados soportan un sufrimiento personal muy importante, sobre todo en el caso de las mujeres, que les lleva a variar de forma negativa su forma de trabajar y su visión de la profesión.

\section{Conflicto de intereses}

Los autores declaran no tener ningún conflicto de intereses.

\section{Agradecimientos}

Los autores quieren agradecer al Departamento de Responsabilidad Civil Profesional de la Agrupación Mutual Aseguradora (AMA), la colaboración prestada para la realización de este trabajo.

\section{Bibliografía}

1. Rubin RJ, Mendelson DN. How much does defensive medicine cost? J Am Health Policy. 1994;4:7-15.

2. Elmore JG, Taplin WE, Cutter GR, D’Orsi CJ, Hendrick RE, Abraham LA, et al. Does litigation influence medical practice? Radiology. 2005;236:37-46.

3. Summerton N. Positive and negative factors in defensive medicine: a questionnaire study of general practitioners. BMJ. 1995;310:27-9.

4. Studdert DM, Mello MM, Sage WM, DesRoches CM, Peugh J, Zapert $\mathrm{K}$, et al. Defensive medicine among high-risk specialist physicians in a volatile malpractice environment. JAMA. 2005;293:2609-17.

5. Hiyama T, Yoshihara $M$. Defensive medicine practices among gastroenterologist in Japan. World J Gastroenterol. 2006;12:7671-5.

6. Christensen JF, Levinson W, Dunn PM. The heart of darkness: the impact of perceived mistakes on physicians. J Gen Intern Med. 1992;7:424-31.

7. Newman MC. The emotional impact of mistakes on family physicians. Arch Fam Med. 1996;5:71-5.

8. Mello MM, Studdert DM, DesRoches CM, Peugh J, Zapert K, Brennan TA, et al. Effects of a malpractice crisis on specialist supply and patients access to care. Ann Surg. 2005;242:621-8.

9. Mello MM, Kelly CN. Effects of a professional liability crisis on residents' practice decisions. Obstet Gynecol. 2005; 105:1287-95.

10. Robinson $\mathrm{P}, \mathrm{Xu} X$, Keeton $\mathrm{K}$. The impact of medical legal risk on obstetrician-gynaecologist supply. Obstet Gynecol. 2005;105:1296-302. 